\title{
Influence of Local Environments in Pores of Different Size on the Catalytic Liquid-Phase Oxidation of D-Glucose by Au Nanoparticles Supported on Nanoporous Carbon
}

Milena Perovic, Nadezda V. Tarakina, Jan Philipp Hofmann, and Martin Oschatz*

Cite This: ACS Appl. Nano Mater. 2020, 3, 7695-7703

Read Online

ACCESS | Lill Metrics \& More | 回 Article Recommendations | sl Supporting Information

ABSTRACT: Inorganic nanoparticles (NPs) dispersed on the surface of porous support materials play a dominant role in heterogeneous catalysis. Especially in liquid-phase catalysis, support materials may lead to nanoconfinement of solvent molecules, and the solute and can thus influence their local concentration and structure. This further affects the surface coverage of nanoparticles with reactants and other species, but these influences remain poorly understood-in particular under experimental conditions. Nanoconfinement effects are of particular importance in reactions such as liquid-phase oxidation with oxygen, i.e., when one reactant is a gas that has to be dissolved before reaching the NP surface. The significant influence of the pore structure of carbon materials on the catalytic activity of gold

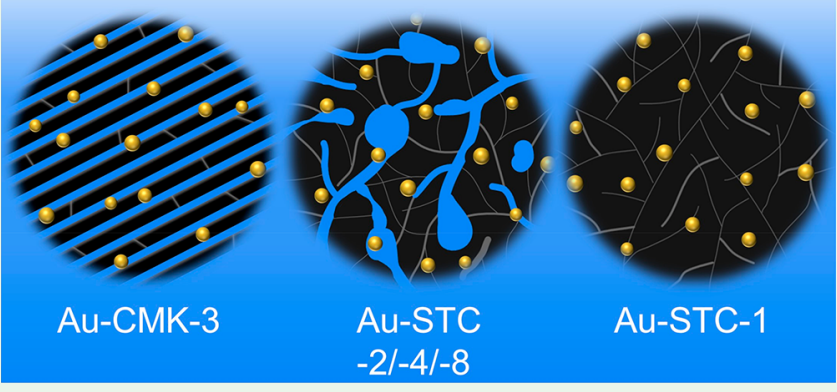
nanoparticles (AuNPs) with nearly similar size (4.1-4.7 nm) is demonstrated in this study. Experimental results on the oxidation of D-glucose with molecular oxygen in aqueous solution show that the "apparent catalytic activity" of AuNPs is a function of the carbon pore size and geometry. The architecture of the carbon pore size is determining the local concentration of reactants. Nanoconfinement of water in carbon nanopores can lead to enhanced solubility of reactants and therefore to their higher local concentration in proximity to the catalytically active sites. In contrast to purely microporous carbon support with the less wettable internal surface without any detectable catalytic activity, AuNPs supported on mesoporous carbons show a much higher metal time yield between 3.8 and $60.6 \mathrm{~mol}_{\mathrm{Glc}} \mathrm{min}^{-1} \mathrm{~mol}_{\mathrm{Au}}{ }^{-1}$, depending on volume and geometry of the mesopores.

KEYWORDS: nanoporous carbons, heterogeneous catalysis, gold nanoparticles, glucose oxidation, confinement, mesopores

\section{INTRODUCTION}

The working principles of catalysis on the surface of nanoparticles (NPs) in a given reaction are determined by the adsorption state and adsorption ratios of all different species present in the distance of a few nanometers, that is, in direct contact with the local active surface. ${ }^{1-4}$ The intrinsic adsorption properties of NPs of given chemical composition (and with that their catalytic properties) mainly depend on the particle size and the strength of interaction with the potentially used porous support material. ${ }^{5}$ Likewise, the properties of the adsorbed (activated) species also depend on both the intrinsic properties of NPs, such as size, exposed facets, surface strain, and electronic states/band structure, and the properties of the fluid phase itself. 6,7 The effect that the NP size has on the adsorption enthalpies and, in consequence, on the catalytic activity can in many cases be described by a volcano-type relationship. 8,9 Furthermore, the structure of the fluid phase surrounding a NP depends on the particle size, as well. For instance, a $2 \mathrm{~nm}$ gold nanoparticle (AuNP) will have a different solvation shell than a $4 \mathrm{~nm}$ one. This is of particular importance for liquid-phase reactions because the local concentration of reactants not only depends on the external factors such as bulk concentration, temperature, or steering rate but also on the molecular orientation of the solvent molecules and other factors such as the local $\mathrm{pH}$ value. In this regard, the properties of particles do not only influence their intrinsic adsorption behavior but may likely also affect the properties of the surrounding phase.

In heterogeneous catalysis, NPs are most often dispersed over support materials with high surface area, which stabilize them and prevent or slow down agglomeration and particle growth throughout the reaction. ${ }^{10-12}$ The chemical and/or electronic interaction between the NPs and the support is known to have a crucial influence on the electronic state of the

Received: May 12, 2020

Accepted: July 10, 2020

Published: July 10, 2020 
NPs and thus on their catalytic properties. ${ }^{13-15}$ The influence of the support porosity on the NPs, and therefore on the catalytic activity, has also been widely studied. ${ }^{16,17}$ However, the possible influence of the nanoporous supports on the properties of the surrounding fluid phase is often neglected. It can be expected that confinement and surface wetting effects occurring in such porous materials have a significant influence on the overall catalytic process. When one reactant in a liquid phase reaction is a gas, its dissolution in the liquid medium is a crucial contribution. As the surface coverage of the catalytically active NPs with reactants is determining their activity and selectivity, the solubility of the gas and reactant in the solvent and the accessibility of the solvent and reactants to the catalyst particles play an important role in heterogeneous catalysis. In general, the solubility of gases in a given liquid changes significantly under pore confinement, as compared to the bulk, in response to capillary pressure and changed molecular orientations. $^{18}$ This effect is influenced by the chemical structure of the pore walls and by the pore size and geometry. For example, a pore in close proximity to a nanoparticle that is too small or pores that are blocked by pores that are too small to be wetted with solvent molecules will not contribute to the enrichment of reactants on the surface of the NPs. In contrast, a NP supported on a material with pores that can be wetted and has strong interaction with the solvent molecules experiences the effects of such pore confinement and is likely surrounded by a phase with a higher concentration of reactants as compared to their concentration in the bulk solution. This change of the "apparent catalytic activity" can be a fundamental principle and an internal regulation screw to influence catalytic processes. From this perspective, NPs of similar size should show different catalytic properties when confined in or surrounded by pores of different sizes provided by a support material with a given chemical structure.

A proof of concept is reported here by using the oxidation of D-glucose (Glc) to gluconic acid in water with molecular oxygen as a model reaction. ${ }^{19-24}$ The catalysts used are AuNPs supported on porous carbon materials of different pore size and geometry. ${ }^{25-27}$ Although the AuNP size, AuNP loadings, and oxidation state as well as the chemical properties of the support are comparable in all cases, the $\mathrm{Au}-\mathrm{C}$ catalysts show significant differences in catalytic activity, depending on pore sizes and geometries of the carbon materials. The influence of these parameters is clearly reflected by the metal time yield (MTY), which ranges from no detectable activity for a microporous salt-templated carbon (STC) support to 3.9 $\mathrm{mol}_{\mathrm{Glc}} \min ^{-1} \mathrm{~mol}_{\mathrm{Au}}^{-1}$ for the least active mesoporous catalyst and up to $60.6 \mathrm{~mol}_{\mathrm{Glc}} \mathrm{min}^{-1} \mathrm{~mol}_{\mathrm{Au}}{ }^{-1}$ for the most active ordered mesoporous catalyst. Even more notable is the difference between the two most active catalysts, where highly mesoporous Au-STC-8 with an SSA $\mathrm{BET}$ of almost $2500 \mathrm{~m}^{2} \mathrm{~g}^{-1}$ but disordered mesopores has a MTY of $30.2 \mathrm{~mol}_{\mathrm{Glc}} \mathrm{min}^{-1}$ $\mathrm{mol}_{\mathrm{Au}}{ }^{-1}$ and ordered mesoporous Au-CMK-3 with SSA $\mathrm{BET}_{\text {of }}$ around $1200 \mathrm{~m}^{2} \mathrm{~g}^{-1}$ reaches $60.6 \mathrm{~mol}_{\mathrm{Glc}} \mathrm{min}^{-1} \mathrm{~mol}_{\mathrm{Au}}{ }^{-1}$.

\section{EXPERIMENTAL SECTION}

Synthesis of STCs. STCs were synthesized by a modified procedure reported by Yan et al. ${ }^{27} \mathrm{ZnCl}_{2}$ was employed as the salt template for the synthesis of STCs. In a typical process, $5 \mathrm{~g}$ of sucrose was dissolved in $15 \mathrm{~mL}$ of water, and a certain amount of $\mathrm{ZnCl}_{2}$ was dissolved in another $15 \mathrm{~mL}$ of water. The prepared solutions were mixed, and $0.55 \mathrm{~g}$ of concentrated sulfuric acid was added. The mixture was transferred to a Petri dish, dried at $100{ }^{\circ} \mathrm{C}$ for $6 \mathrm{~h}$, and subsequently heated to $160^{\circ} \mathrm{C}$ and kept for $6 \mathrm{~h}$. The amount of $\mathrm{ZnCl}_{2}$ is determined by the mass ratio of the salt template to sucrose regarding different STCs (i.e., in STC- $X, X$ stands for the mass ratio of $\mathrm{ZnCl}_{2}$ /sucrose). The mixture was then transferred to a horizontal tubular furnace for carbonization at $900{ }^{\circ} \mathrm{C}$ for $2 \mathrm{~h}$ under $\mathrm{N}_{2}$ flow (heating rate: $60^{\circ} \mathrm{C} \mathrm{h}^{-1}$ ). Afterward, the salt residues were washed by stirring the materials in $1 \mathrm{M} \mathrm{HCl}$ for $72 \mathrm{~h}$, followed by filtration and washing with water.

Synthesis of SBA-15 and CMK-3. The hexagonal ordered silica template SBA-15 was synthesized by dissolving $33.4 \mathrm{~g}$ of the triblock copolymer Pluronic P123 (EO $\mathrm{EO}_{20} \mathrm{PO}_{70} \mathrm{EO}_{20}$, Sigma-Aldrich) in $606 \mathrm{~g}$ of deionized water and $19.3 \mathrm{~g}$ of concentrated aqueous hydrochloric acid solution overnight at $35{ }^{\circ} \mathrm{C}$ in a $1000 \mathrm{~mL}$ polypropylene bottle under intense stirring. Then, $71.8 \mathrm{~g}$ of tetraethyl orthosilicate (TEOS, $98 \%$, Sigma-Aldrich) was added to the solution, and the mixture was stirred at $35{ }^{\circ} \mathrm{C}$ for another $24 \mathrm{~h}$. The white suspension was then transferred to a Teflon-lined autoclave and hydrothermally treated at $130{ }^{\circ} \mathrm{C}$ for $24 \mathrm{~h}$ followed by filtration and washing with $\sim 1000 \mathrm{~mL}$ of deionized water/ethanol (1:1 by volume). For complete removal of the structure-directing agent, the SBA- 15 was calcined at $550{ }^{\circ} \mathrm{C}$ for 5 $\mathrm{h}$ in a muffle furnace under an air atmosphere (heating rate: $60^{\circ} \mathrm{C}$ $\left.\mathrm{h}^{-1}\right){ }^{28}$

Ordered mesoporous carbon CMK-3 was synthesized by impregnating $4 \mathrm{~g}$ of SBA-15 with a $20 \mathrm{~mL}$ aqueous solution of $5 \mathrm{~g}$ of sucrose, to which was added $0.56 \mathrm{~g}$ of $96 \%$ sulfuric acid. Polymerization of the carbohydrate was achieved by heating the mixture to $100{ }^{\circ} \mathrm{C}$ for $6 \mathrm{~h}$ followed by subsequent heating to $160^{\circ} \mathrm{C}$ for another $6 \mathrm{~h}$. Complete infiltration of the template pores was achieved by repeating the procedure described above with a $20 \mathrm{~mL}$ aqueous solution of $3.2 \mathrm{~g}$ of sucrose, to which was added $0.36 \mathrm{~g}$ of $96 \%$ sulfuric acid, again followed by heating to 100 and $160{ }^{\circ} \mathrm{C}$. Carbonization was performed under a flowing $\mathrm{N}_{2}$ atmosphere in a horizontal tubular furnace. The material was heated to $900{ }^{\circ} \mathrm{C}$ (heating rate: $150{ }^{\circ} \mathrm{C} \mathrm{h}^{-1}$ ) and dwelled for $2 \mathrm{~h}$. Silica removal was achieved by refluxing the carbonized composite material in sodium hydroxide solution $\left(400 \mathrm{~mL}, 5 \mathrm{~mol} \mathrm{~L}^{-1}\right)$ overnight. After filtration and washing with large amounts of ethanol, the CMK-3 material was dried at $60{ }^{\circ} \mathrm{C}$. The carbon yield was $\sim 2.2 \mathrm{~g}^{25}$

All of the synthesized carbons (STCs and CMK-3) were treated under a flow of reducing atmosphere $\left(5 \% \mathrm{H}_{2}\right.$ in $\left.\mathrm{N}_{2}\right)$ for $2 \mathrm{~h}$ at $600{ }^{\circ} \mathrm{C}$ (heating rate: $240{ }^{\circ} \mathrm{C} \mathrm{h}^{-1}$ ).

Synthesis of Catalysts. The colloidal gold dispersion was synthesized by a modified Turkevich method. ${ }^{29} 29 \mathrm{~mL}$ of $0.2 \mathrm{wt} \%$ $\mathrm{HAuCl}_{4} \cdot 3 \mathrm{H}_{2} \mathrm{O}$ ( $49 \mathrm{wt} \% \mathrm{Au}$ ) was added to $500 \mathrm{~mL}$ of deionized water while stirring. Then $1 \mathrm{wt} \%$ sodium citrate aqueous solution (11.6 $\mathrm{mL}$ ) was added. After $30 \mathrm{~s}, 5.80 \mathrm{~mL}$ of freshly prepared sodium borohydride solution ( $17 \mathrm{mg}$ of $\mathrm{NaBH}_{4}$ in $10 \mathrm{~mL}$ of ice-cooled $1 \mathrm{wt} \%$ aqueous sodium citrate solution) was added to the mixture.

Gold nanoparticles were deposited on carbon supports by immersing $500 \mathrm{mg}$ of the carbon into $91.1 \mathrm{~mL}$ of colloidal gold dispersion, followed by sonication for $2 \mathrm{~h}$. The catalyst was separated by centrifugation at $4000 \mathrm{rpm}$ for $20 \mathrm{~min}$, washed three times with deionized water, and dried overnight in a vacuum oven at $60^{\circ} \mathrm{C}$. The catalysts are labeled as Au-STCs and Au-CMK-3.

Characterization of Support Materials and Catalysts. Prior to all of the physisorption measurements, the samples were outgassed under vacuum at $150{ }^{\circ} \mathrm{C}$ for $20 \mathrm{~h} . \mathrm{N}_{2}$ physisorption experiments were performed at $-196{ }^{\circ} \mathrm{C}$ on a Quadrasorb apparatus (Quantachrome Instruments, USA). Specific surface areas (SSA) of the materials are calculated by using the multipoint Brunauer-Emmett-Teller (BET) model in the relative pressure range $0.05-0.2$. The total pore volumes $\left(V_{\mathrm{t}}\right)$ were determined at $p / p_{0}=0.99$. The pore size distributions are calculated by using the quenched solid density functional theory (QSDFT) method for nitrogen on carbon with slit/cylindrical/ spherical pores at $-196{ }^{\circ} \mathrm{C}$, adsorption branch kernel, integrated into the QuadraWin 5.11 analysis software (Quantachrome). Micropore volumes $\left(V_{\text {micro }}\right)$ were calculated by using the DFT method from the cumulative pore volumes at a diameter of $2 \mathrm{~nm}$.

High-resolution transmission electron microscopy (HRTEM) was performed by using a JEOL ARM 200F microscope operated at 200 
$\mathrm{kV}$. TEM was performed on a Zeiss912 Omega operated at $120 \mathrm{kV}$. Prior to analysis, the samples were dispersed in ethanol and sonicated for $5 \mathrm{~min}$. Several droplets of dispersions were cast onto TEM copper grids with a holey carbon film and dried at room temperature.

$\mathrm{X}$-ray photoelectron spectroscopy (XPS) was performed on a Thermo Scientific K-Alpha spectrometer equipped with an $\mathrm{Al} \mathrm{K \alpha}$ anode ( $h v=1486.7 \mathrm{eV} ; 400 \mu \mathrm{m}$ spot size). Elemental compositions were determined by using survey scans over a range of $1350-0 \mathrm{eV}$ with a step size of $1.0 \mathrm{eV}$ and a pass energy of $200 \mathrm{eV}$. Quantitative characterization of the presence of $\mathrm{C}, \mathrm{O}$, and $\mathrm{Au}$ was accomplished with high-resolution XPS region scans with a step size of $0.1 \mathrm{eV}$ and a pass energy of $50 \mathrm{eV}$. Scanning ranges were 295-280, 545-525, and 99-79 eV, respectively. Shipping and handling were done in ambient conditions without further precautions. The samples were measured in a $\mathrm{Cu}$ metal powder sample holder without the use of conductive tape. Charge compensation was achieved by an electron flood gun. Binding energy calibration was performed by setting the $\mathrm{C} 1 \mathrm{~s}$ binding energy of the graphitic $\mathrm{sp}^{2}$ carbon to $284.5 \mathrm{eV}$.

Powder X-ray diffraction (PXRD) patterns were recorded on a Bruker D8 Advance diffractometer equipped with a scintillation counter detector using $\mathrm{Cu} \mathrm{K} \alpha$ radiation $(\lambda=0.1518 \mathrm{~nm})$ in the $2 \theta$ range $3^{\circ}-90^{\circ}$ with a step size of $0.02^{\circ}$ and counting time of $1 \mathrm{~s}$ per step.

Raman spectra were recorded by using a Witec Raman microscope operating with an objective (Nikon, $10 \times / 0.25, \infty /-W D$ 6.1) and an excitation wavelength of $532 \mathrm{~nm}$ with an intensity of $3.5 \mathrm{~mW}$ and accumulations of 100 scans with $10 \mathrm{~s}$ per scan. Deconvolution of the spectra was performed by assuming mixed Gaussian/Lorentzian peaks to describe both the main D- and G-bands and the two bands with lower intensity, $\mathrm{A}$ and $\mathrm{D}^{2}$, positioned at 1500 and $1170 \mathrm{~cm}^{-1}$, respectively. The fit was performed by using OriginPro 2019. The parameters retained were the full width at half-maximum (FWHM) of the D-band and the ratio of the peak heights $\left(I_{\mathrm{D}} / I_{\mathrm{G}}\right)$.

Thermogravimetric analyses (TGA) were performed by using a thermo-microbalance TG 209 F1 Libra (Netzsch, Selb, Germany). A platinum crucible was used for the measurements of $10 \pm 1 \mathrm{mg}$ of samples in synthetic air flow. The sample was heated to $1000{ }^{\circ} \mathrm{C}$ with a heating rate of $10{ }^{\circ} \mathrm{C} \mathrm{min}-1$. The data were recorded and analyzed by the Proteus (6.0.0) and software package.

Inductively coupled plasma optical emission spectrometry (ICPOES) was conducted by using a Horiba Ultra 2 instrument equipped with photomultiplier tube detection. The content of the gold in the catalysts was determined by analyzing the solution after deposition on the carbon support. A metal leaching test was conducted by analyzing the catalytic reaction solution after the reaction.

Catalytic Activity Tests. The catalytic oxidation of D-glucose was monitored via a potentiometric titrator (TitroLine 6000/7000) device with the functionality of a TITRONIC piston buret. The reaction was performed with $50 \mathrm{~mL}$ of $0.1 \mathrm{M}$ D-glucose and $50 \mathrm{mg}$ of $1 \mathrm{wt} \% \mathrm{Au}$ on a C catalyst at $45{ }^{\circ} \mathrm{C}, \mathrm{pH}=9$, under an oxygen flow of $\approx 250 \mathrm{~mL}$ $\min ^{-1}$ and stirring at $800 \mathrm{rpm}$. The $\mathrm{pH}$ of the solution was kept constant at $\mathrm{pH}=9$ by automatic titration with $1 \mathrm{M}$ aqueous $\mathrm{NaOH}$ solution. The catalytic activity was evaluated from the slope of the titration curve, in the range between 1 and $3 \mathrm{~mL}$ of added $\mathrm{NaOH}$, which corresponds to the conversion between $20 \%$ and $60 \%$ of Dglucose.

Control catalytic experiments without the oxygen flow were performed by using selected mesoporous catalysts: Au-CMK-3, AuSTC-8, and Au-STC-2. The dispersion of a catalyst in water $(50 \mathrm{mg}$ in $10 \mathrm{~mL}$ ) was saturated with oxygen under a flow of $\approx 250 \mathrm{~mL} \mathrm{~min}^{-1}$ for $7 \mathrm{~min}$. Then the oxygen flow was stopped, and D-glucose solution $(0.125 \mathrm{M})$ was added to the mixture to obtain a final concentration of $0.1 \mathrm{M}$. Finally, monitoring the reaction with the titrator described above was started. The atmosphere above the reaction mixture was purged with nitrogen.

The products of the D-glucose oxidation reaction were analyzed by nuclear magnetic resonance spectroscopy (NMR) on a Bruker Ascend $400(400 \mathrm{MHz})$ spectrometer. Chemical shifts $\delta$ are reported in ppm and are adjusted to internal standards of the residual proton signal of the deuterated solvent $\left(\mathrm{D}_{2} \mathrm{O}: 4.79 \mathrm{ppm}\right.$ for $\left.{ }^{1} \mathrm{H}\right)$. The spectra were measured at room temperature. Having symmetrical signals, the center of this signal is given and for multiplets the area. Thereby the following characterization was used: $\mathrm{s}=$ singlet, $\mathrm{sbr}=$ singlet broad, $\mathrm{d}$ $=$ doublet, $\mathrm{t}=$ triplet, $\mathrm{q}=$ quartet, $\mathrm{m}=$ multiplet or combinations like $\mathrm{dd}=$ doublet of doublet, or $\mathrm{dt}=$ doublet of triplet. Coupling constants $(J)$ are given in hertz. Data were evaluated by using MestReNova v.12.0.4-22023 software. For ${ }^{1} \mathrm{H}$ NMR spectra, the correlation of the signals was done by the multiplicities. $2 \mathrm{D}$ spectra were recorded by heteronuclear single-quantum correlation (HSQC) as well as heteronuclear multiple-bond correlation (HMBC) spectroscopy. ${ }^{30}$

\section{RESULTS AND DISCUSSION}

Salt-templated carbons (STCs) have been used as model supports because their porosity can be tuned over a wide range by simply adjusting the ratio between porogen and carbon source. ${ }^{26,27} \mathrm{ZnCl}_{2}$ and sucrose have been used as a porogen and as carbon precursor, respectively. After the carbonization of the carbohydrate-salt mixture, the material was thoroughly washed with $\mathrm{HCl}$ solution to remove the remaining zinc species. Removal of the salt template led to the formation of micropores and mesopores. STC materials are labeled as STC$X$, with $X$ representing the mass ratio of $\mathrm{ZnCl}_{2}$ to sucrose. Another material investigated is ordered mesoporous carbon (CMK-3), which has been obtained by nanocasting of silica template (SBA-15) with sucrose, followed by carbonization and subsequent template removal (Scheme 1). ${ }^{25,28,31}$

\section{Scheme 1. Synthesis Procedure of CMK-3 and STC Carbon} Supports

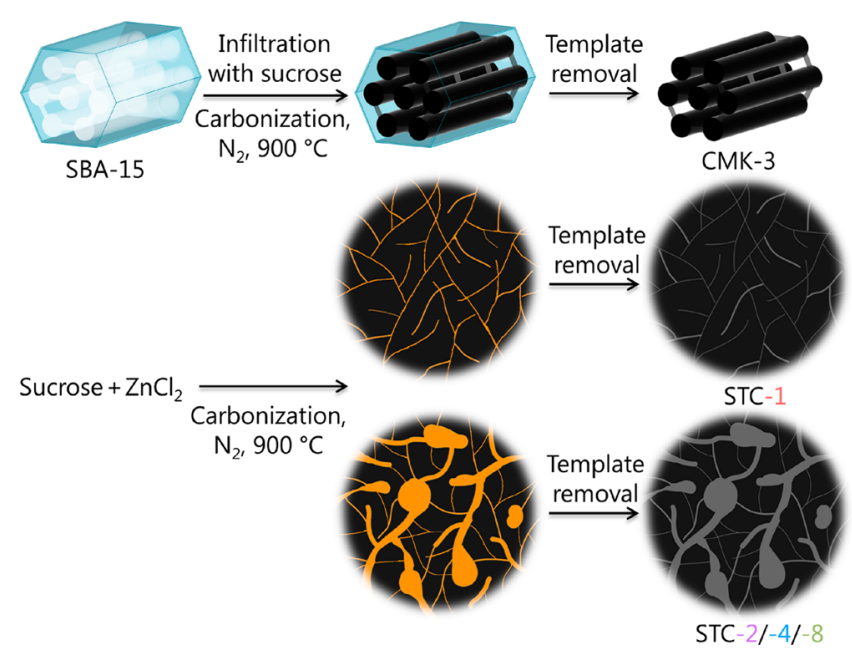

$\mathrm{N}_{2}$ physisorption experiments $\left(-196^{\circ} \mathrm{C}\right)$ were performed to investigate the pore structures of the prepared carbons (Figure 1 and Table 1). STC-1 displays a type I(a) isotherm according to the IUPAC classification as it is typical for solids with mainly narrow micropores. ${ }^{32}$ The type I(b) isotherm of STC-2 and the presence of a narrow hysteresis loop indicate that there is a small amount of mesopores present in this material in addition to the micropores. STC-4 and STC-8 contain larger disordered mesopores and thus show type IV(a) isotherms as well as hysteresis loops of $\mathrm{H} 4$ type in the relative pressure range between 0.5 and 0.9 . CMK-3 also reveals a type $\operatorname{IV}(\mathrm{a})$ isotherm but with a type $\mathrm{H} 2$ (a) hysteresis loop in the $p / p_{0}$ range of $0.4-0.7$, demonstrating its ordered mesoporous nature. The pore size distribution (PSD) of STCs and CMK-3 was further analyzed by using the quenched-solid density functional theory (QSDFT, adsorption branch kernel) for $\mathrm{N}_{2}$ 

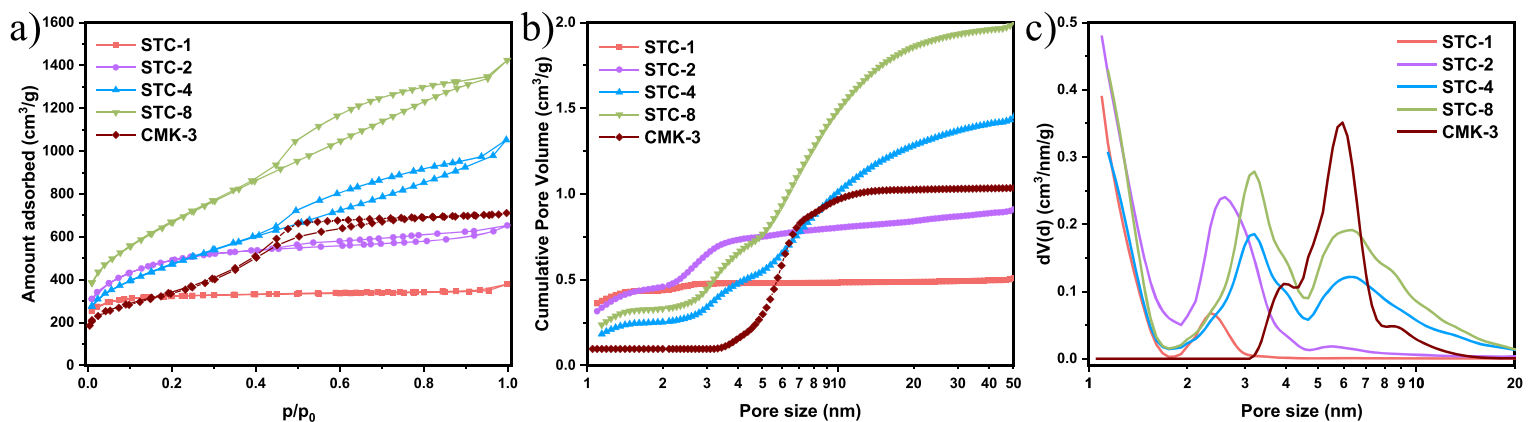

Figure 1. (a) $\mathrm{N}_{2}$ physisorption isotherms (at $-196^{\circ} \mathrm{C}$ ) with the corresponding (b) cumulative and (c) differential pore size distribution plots calculated with QSDFT ( $\mathrm{N}_{2}$ on carbons with slit/cylindrical/spherical pores at $77 \mathrm{~K}$, adsorption branch kernel) of STCs and CMK-3.

Table 1. Specific Surface Area (SSA $\left.{ }_{\mathrm{BET}}\right)$ Calculated via the BET Equation, Total Pore Volume $\left(V_{\mathrm{t}}\right)$, DFT Micropore Volume $\left(V_{\text {micro }}\right)$, and DFT Mesopore Volume $\left(V_{\text {meso }}\right)$ Obtained from $N_{2}$ Physisorption Isotherms (at $\left.-196{ }^{\circ} \mathrm{C}\right), I_{\mathrm{D}} / I_{\mathrm{G}}$ Ratio, and FWHM of the D-Band of STCs and CMK-3 Obtained by Raman Spectroscopy

$\begin{array}{lcccccc}\text { sample } & \mathrm{SSA}_{\mathrm{BET}}\left[\mathrm{m}^{2} \mathrm{~g}^{-1}\right] & V_{\mathrm{t}}\left[\mathrm{cm}^{3} \mathrm{~g}^{-1}\right] & V_{\text {micro }}\left[\mathrm{cm}^{3} \mathrm{~g}^{-1}\right] & V_{\text {meso }}\left[\mathrm{cm}^{3} \mathrm{~g}^{-1}\right] & I_{\mathrm{D}} / I_{\mathrm{G}} & \text { FWHM of the D-band }\left[\mathrm{cm}^{-1}\right] \\ \text { STC-1 } & 1210.2 & 0.59 & 0.43 & 0.11 & 1.8 & 213 \\ \text { STC-2 } & 1748.5 & 1.01 & 0.45 & 0.52 & 1.1 & 153 \\ \text { STC-4 } & 1711.3 & 1.63 & 0.25 & 1.27 & 1.1 & 159 \\ \text { STC-8 } & 2429.4 & 2.21 & 0.33 & 1.75 & 1.3 & 155 \\ \text { CMK-3 } & 1208.0 & 1.10 & 0.09 & 1.00 & 1.0 & 171\end{array}$

adsorbed on carbon with slit/cylindrical/spherical pore shape (Figure 1b). STCs have comparable micropore volumes in the range $0.25-0.45 \mathrm{~cm}^{3} \mathrm{~g}^{-1}$, whereas CMK-3 shows a much lower micropore volume of $0.09 \mathrm{~cm}^{3} \mathrm{~g}^{-1}$. In the range from STC-1 to STC-8, the mesopore volume gradually increases with increased salt template content (from $0.11 \mathrm{~cm}^{3} \mathrm{~g}^{-1}$ for STC1 to $1.75 \mathrm{~cm}^{3} \mathrm{~g}^{-1}$ for STC-8). In the case of silica-templated CMK-3 the mesopore volume is $1.00 \mathrm{~cm}^{3} \mathrm{~g}^{-1}$. The latter shows a narrow pore size distribution centered around a diameter of 6 $\mathrm{nm}$ (Figure 1b,c). On the other hand, all of the mesoporous STCs show an $\mathrm{N}_{2}$ uptake over a wider range of relative pressures due to their rather broad pore size distribution. All of the materials provide high (multipoint) Brunauer-EmmettTeller specific surface areas $\left(\mathrm{SSA}_{\mathrm{BET}}\right)$ in the range $\sim 1200-$ $2500 \mathrm{~m}^{2} \mathrm{~g}^{-1}$. SSA $\mathrm{BET}$ is gradually increasing in the range from STC- 1 to STC-8, and CMK-3 has a SSA $A_{\mathrm{BET}}$ that is comparable to that of STC-1.

Thermogravimetric analysis (TGA) of the carbon materials under synthetic air show a small ash content ( $<3 \mathrm{wt} \%)$ above $600{ }^{\circ} \mathrm{C}$ and no remaining mass above $500{ }^{\circ} \mathrm{C}$ for CMK-3 (Figure S1, Supporting Information). This shows the absence of significant amounts of inorganic residuals and thus the successful removal of $\mathrm{Zn}$ species and $\mathrm{SiO}_{2}$ template from the STCs and CMK-3, respectively.

Raman spectra of the carbon supports were fitted by using a four-band model with mixed Gaussian/Lorentzian peaks (Figure S2). ${ }^{33,34}$ All of the carbons, independent of the templating method, show typical spectra of the disordered carbon nanomaterials, with the D-band at $\sim 1340 \mathrm{~cm}^{-1}$ and the G-band at $\sim 1595 \mathrm{~cm}^{-1}$. The D-band (disordered) originates from the breathing mode of $\mathrm{sp}^{2}$-hybridized carbon atoms in aromatic rings in the proximity of an edge or point defect. The width of the D-band points to the degree of structural ordering and size distribution of $\mathrm{sp}^{2}$ carbon rings. ${ }^{35}$ The full width at half-maximum (FWHM) of the D-band for all carbon supports is relatively wide, indicating a large number of structurally disordered units within these materials (Table 1). The G-band (graphite-like) originates from vibrations of all $\mathrm{sp}^{2}$ carbons organized in chains or rings. The peak intensity ratio of the Dand G-band $\left(I_{\mathrm{D}} / I_{\mathrm{G}}\right)$ is a useful value for assessing the level of carbon ordering in such nanoporous materials. With the exception of STC-1, which has a slightly more disordered structure dominated by narrow pores, the values of $I_{\mathrm{D}} / I_{\mathrm{G}}$ and the FWHM of the D-band for all STCs and CMK-3 are in the same range $\left(153-171 \mathrm{~cm}^{-1}\right)$. This implies that the materials have a comparable degree of aromatization. No distinct differences in their redox potential are expected which would affect the electronic state or chemical properties of supported NPs.

A series of catalysts were synthesized by depositing a nominal loading of $1 \mathrm{wt} \%$ of AuNPs on the carbon supports (denoted as Au-STC- $X$ and Au-CMK-3). X-ray powder diffraction (XRD) measurements of the catalysts show typical amorphous carbon patterns together with four peaks corresponding to Bragg reflections of the (111), (200), (220), and (311) planes of the face-centered cubic lattice of gold (Figure 2). The presence of broad peaks implies that gold

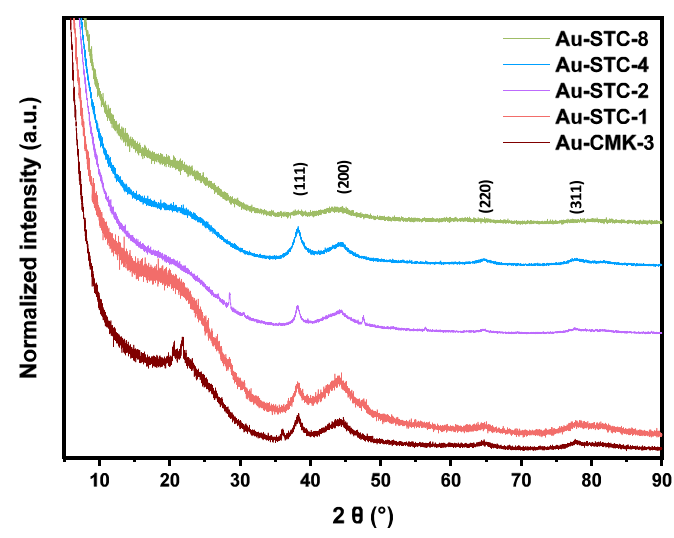

Figure 2. XRD patterns of Au-STCs and Au-CMK-3 catalysts. 
is indeed deposited on the carbons as nanoparticles. The low intensity of $\mathrm{Au}$ reflections in Au-STC-8 could be ascribed to the high background noise of highly porous carbon support STC-8.

Figure 3 presents transmission electron microscopy (TEM) images of the carbon-supported catalysts together with the
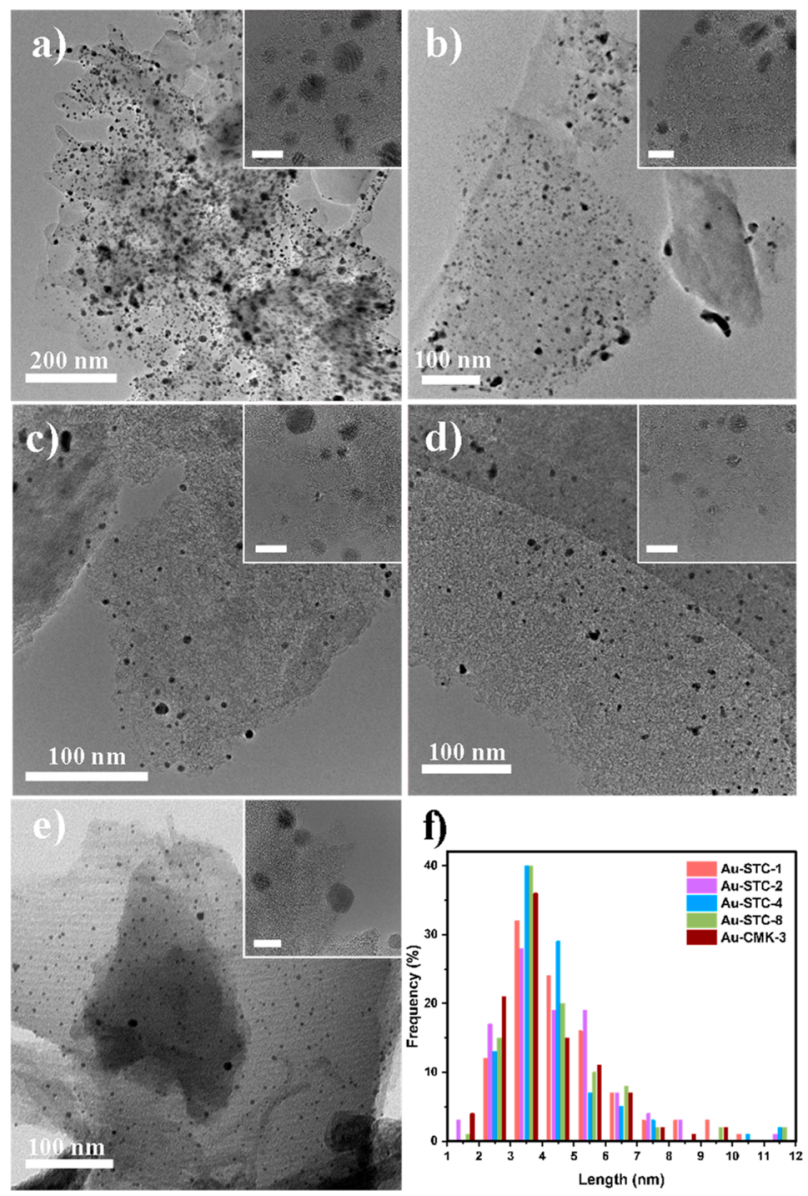

Figure 3. TEM images of catalysts: (a) Au-STC-1, (b) Au-STC-2, (c) Au-STC-4, (d) Au-STC-8, (e) Au-CMK-3, and (f) corresponding AuNP size distributions. Insets show HRTEM images of the catalysts; the length of the scale bar is $10 \mathrm{~nm}$.

corresponding particle size distributions. In the series of AuSTCs, the morphology of the carbon support changes from a rather dense structure for the microporous Au-STC-1 to a gradually more porous appearance in the series from Au-STC2 to $\mathrm{Au}-\mathrm{STC}-8$. TEM of Au-CMK-3 (Figure 3e) reveals its mesoporous system with ordered carbon nanorods. Despite the different porosities of the support materials, the deposition of AuNPs resulted in comparable particle size distributions (Figure 3f). The average AuNP sizes of the catalysts span over a rather narrow range from $4.1 \mathrm{~nm}$ for Au-CMK-3 to $4.7 \mathrm{~nm}$ for Au-STC-1 (Table 2). The deposition of stabilized AuNPs from dispersion onto the carbon surfaces can be described by a ligand-exchange mechanism. ${ }^{36}$ The citrate stabilizer can be replaced with stabilization of the AuNPs by surface oxygen groups present on the carbon or by formation of donoracceptor couples with the $\pi$-electrons of the supports. TEM images show that particles are mainly inhomogeneously deposited on the external surface of the carbon support. One has to notice that prior to the TEM investigations the particles were washed/centrifuged and then sonicated for electron microscopy sample preparation. Because the pore sizes of the supports (Figure $1 \mathrm{~b}, \mathrm{c}$ ) and the sizes of the AuNPs are in the same range, it is likely that the gold particles cannot easily enter the bulk of large carbon support particles. Ligand exchange from citrate to the surface of porous carbon occurs rapidly, and the speed of migration of the AuNPs into the narrow pores of the support materials is further limited by the presence of stabilizer and solvation shell. Furthermore, it has been shown recently that the surface chemistry of carbon supports with comparable pore architecture (i.e., the strength of the carbon as a ligand for the AuNPs) plays a crucial role in the degree of agglomeration of the particles during deposition. ${ }^{37}$ It is also widely accepted that the surface chemistry of carbon supports plays a crucial role in the catalytic activity of deposited nanoparticles-even when their size is in a comparable range. ${ }^{38,39}$ To minimize this influence, the surface chemistry of all carbon materials under investigation has been adjusted by thermal treatment under reducing atmosphere, the aim of which is to provide similar strength of interactions of the AuNPs with the carbon supports during deposition. The rather hydrophobic surface atomic structure of carbonaceous support materials leads to a slower ligand exchange between the citrate stabilizer of the AuNPs and the carbon support. This results in less agglomeration and coalescence and therefore better stabilization of smaller AuNPs. ${ }^{37}$

X-ray photoelectron spectroscopy (XPS) was employed to characterize the oxidation state and surface amount of gold species. The survey XPS spectra (Figure 4a) of all of the AuSTCs and Au-CMK-3 catalysts reveal the presence of $\mathrm{C}, \mathrm{O}$, and $\mathrm{Au}$. The high-resolution $\mathrm{Au}$ 4f spectra (Figure $4 \mathrm{~b}-\mathrm{f}$ ) display the main $\mathrm{Au} 4 \mathrm{f}_{7 / 2}$ peak at $84.0-84.2 \mathrm{eV}$ with a spinorbit splitting of $3.67 \mathrm{eV}$, which corresponds to metallic $\mathrm{Au}$ species (Table S1). The additional smaller doublet at $\mathrm{Au} 4 \mathrm{f}_{7 / 2}$ that lies at $86.0-86.2 \mathrm{eV}$ could be related to the presence of $\mathrm{Au}^{\delta+}$ or $\mathrm{Au}^{+}{ }^{+40-42}$ The content of gold determined by XPS on the surfaces of the catalyst particles is between 0.3 and 1.4 at.

Table 2. XPS, ${ }^{a}$ ICP-OES Results, Average AuNP Sizes before and after (Values in Parentheses) the D-Glucose Oxidation Reaction, Determined by TEM, and Catalytic Activity for the D-Glucose Oxidation Reaction

\begin{tabular}{|c|c|c|c|c|c|c|}
\hline sample & $\mathrm{C}[\text { at. } \%]_{\mathrm{XPS}}$ & $\mathrm{O}[\text { at. } \%]_{\mathrm{XPS}}$ & $\mathrm{Au}[\text { at. } \%]_{\mathrm{XPS}}$ & $\mathrm{Au}[\mathrm{wt} \%]_{\mathrm{ICP}}$ & av AuNP size $[\mathrm{nm}]_{\mathrm{TEM}}$ & catalytic activity $\left[\mathrm{mol}_{\mathrm{Glc}} \min ^{-1} \mathrm{~mol}_{\mathrm{Au}}^{-1}\right]$ \\
\hline Au-STC-1 & 91 & 5.4 & 1.2 & 0.8 & $4.7 \pm 1.7(4.0 \pm 1.5)$ & 0 \\
\hline $\mathrm{Au}-\mathrm{STC}-2$ & 95 & 3.7 & 0.6 & 1.0 & $4.5 \pm 1.7(4.5 \pm 1.9)$ & 3.9 \\
\hline $\mathrm{Au}-\mathrm{STC}-4$ & 96 & 2.8 & 0.4 & 1.0 & $4.4 \pm 1.6(4.4 \pm 1.8)$ & 12.9 \\
\hline Au-STC-8 & 95 & 2.4 & 1.4 & 0.9 & $4.4 \pm 1.7(3.7 \pm 1.4)$ & 30.2 \\
\hline $\mathrm{Au}-\mathrm{CMK}-3$ & 96 & 2.9 & 0.7 & 1.0 & $4.1 \pm 1.6(4.0 \pm 1.6)$ & 60.6 \\
\hline
\end{tabular}

${ }^{a}$ In addition to the elements in the table, XPS showed the presence of S in following samples: Au-STC-1 (2.4 at. \%), Au-STC-2 (0.5 at. \%), AuSTC-4 (0.4 at. \%), and Au-STC-8 (0.9 at. \%) as well as the presence of $\mathrm{Zn}$ in Au-STC-1 (0.2 at. \%). 

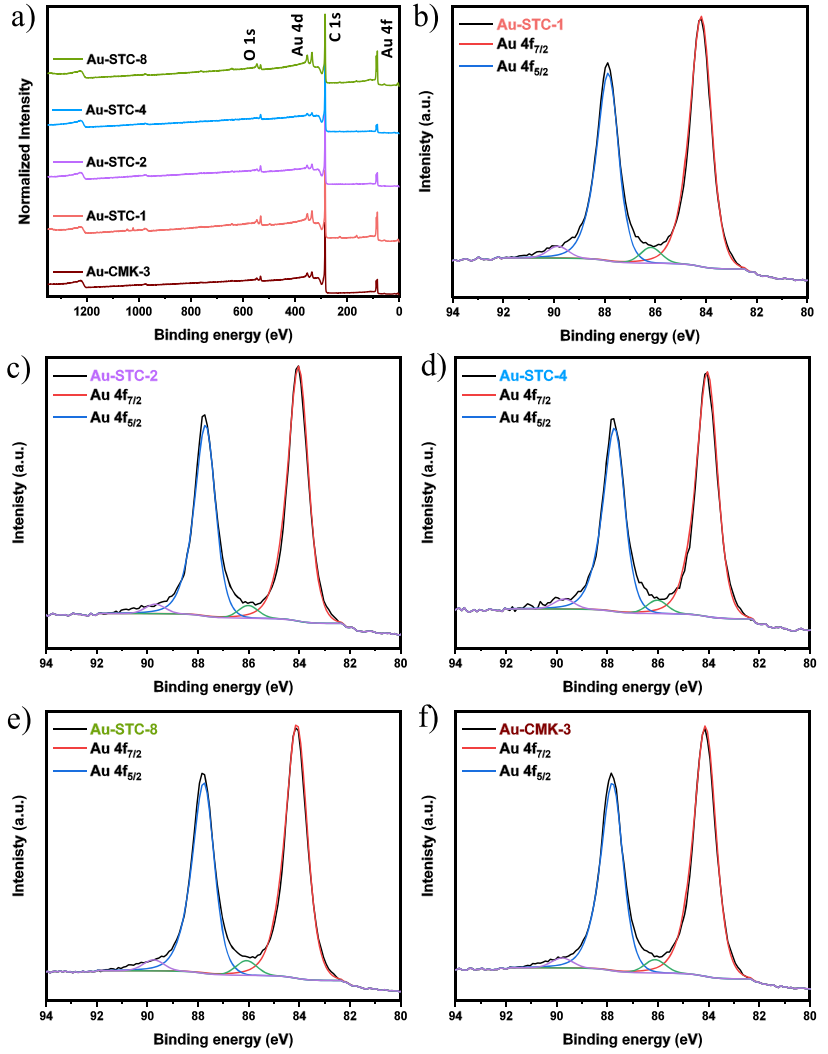

Figure 4. (a) XPS survey scans of the catalysts and Au 4f XPS spectra with fitted components of (b) Au-STC-1, (c) Au-STC-2, (d) AuSTC-4, (e) Au-STC-8, and (f) Au-CMK-3.

$\%$ (Table 2). These values are significantly higher than the bulk $\mathrm{Au}$ content determined by inductively coupled plasma optical emission spectrometry (ICP-OES) (Table 2). This indicates that the AuNPs are mainly located on the external surface of the carbon supports in all cases. C 1s (Figure S3) and O 1s spectra (Figure S4) show comparable shapes and relative contents for the entire series of the catalysts as well. Two types of electron-rich and electron-deficient oxygen species are detected, with binding energies centered at 531.0-531.5 and $532.8-533.3 \mathrm{eV}$, respectively. The content of carbon (expressed through the integrated $\mathrm{C} 1 \mathrm{~s}$ peaks) ranges between 90.8 and 96.4 at. \%, and the oxygen content (expressed through $\mathrm{O} 1 \mathrm{~s}$ peaks) is between 2.4 and 5.4 at. \% (Table 2). There is no obvious correlation between the oxygen contents and the catalytic activity of the carbon supports discussed below (Figure S5). Along with $\mathrm{C}$, O, and Au, XPS of STCsupported catalysts shows the presence of S (0.4-2.4 at. \%), which is due to the usage of sulfuric acid in the synthesis. Because this species remained in the samples after the carbonization under an inert atmosphere, as well as treatment in the reducing atmosphere, it can be deduced that sulfur is stable in carbon materials. In addition, 0.2 at. \% of $\mathrm{Zn}$ is found in Au-STC-1, which originates from the use of $\mathrm{ZnCl}_{2}$ as a porogen.

Characterization of the carbon supports and the gold catalysts reveals that the surface chemistry of all of the samples is comparable. Furthermore, the content and oxidation state of $\mathrm{Au}$ and the average AuNPs sizes are in the same range in all $\mathrm{Au}-\mathrm{STCs}$ and Au-CMK-3 catalysts. Thus, it can be concluded that the performance of these catalysts will be mainly affected by the pore structures of the carbonaceous supports. One would not expect a significant difference in the catalytic activity of these materials in a liquid phase oxidation reaction with molecular oxygen as it is generally accepted that the size of AuNPs and the chemical properties of the support material are the factors with crucial influence.

Catalytic activities of the catalysts were tested by applying the oxidation of D-glucose with molecular oxygen in aqueous solution at $\mathrm{pH}=9$ and $45{ }^{\circ} \mathrm{C}$ as a model reaction. The resulting titration curves with $1 \mathrm{M}$ aqueous $\mathrm{NaOH}$ express the conversion of D-glucose to gluconic acid as a function of time (Figure 5). Conversion is increasing with mesopore volume in

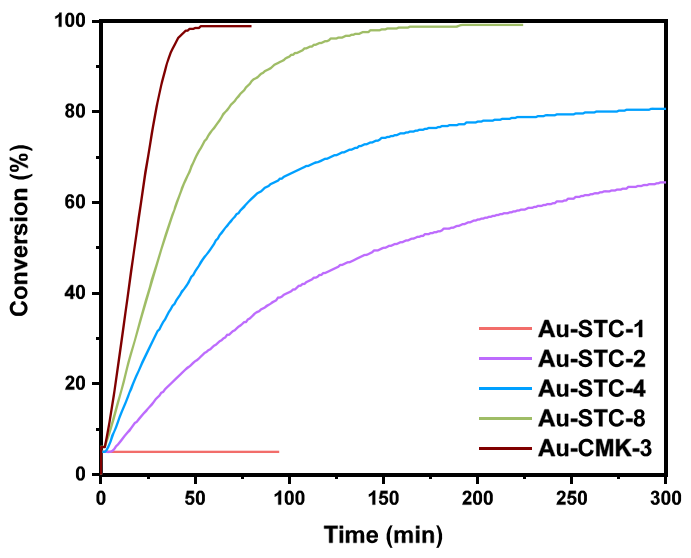

Figure 5. Conversion vs time diagram representing the catalytic activities of the catalysts supported on STCs and CMK-3 for Dglucose oxidation.

the series of Au-STCs, starting from no detectable conversion for Au-STC-1 to full conversion in $189 \mathrm{~min}$ given by reaction with Au-STC-8. The fastest full conversion is accomplished with Au-CMK-3 after $53 \mathrm{~min}$. This corresponds to a remarkable catalytic activity of $60.6 \mathrm{~mol}_{\mathrm{Glc}} \min ^{-1} \mathrm{~mol}_{\mathrm{Au}}{ }^{-1}$ when using Au-CMK-3. In comparison, the least active mesoporous catalyst, Au-STC-2, shows the significantly lower activity of $3.9 \mathrm{~mol}_{\mathrm{Glc}} \mathrm{min}^{-1} \mathrm{~mol}_{\mathrm{Au}}{ }^{-1}$ (Table 2 and Figure S5). TEM investigations of the catalysts after the reaction show no significant changes in the morphology or average AuNP size distribution (Table 2 and Figure S6), and the effects of particle agglomeration during the reaction do not seem to have a crucial influence in all cases. As discussed above, there is indeed a minor difference and trend in the average AuNP sizes ranging between $4.7 \mathrm{~nm}$ for the least active Au-STC-1 and 4.1 $\mathrm{nm}$ for the most active Au-CMK-3. However, although AuNP sizes are a crucial parameter in this reaction, minor differences as present here alone cannot explain differences in MTY that span over more than 1 order of magnitude. Furthermore, AuSTC-4 and Au-STC-8 have similar average AuNP sizes but differ significantly in their activity (Table 2 and Figure S7).

The results suggest that for the series of STC-supported catalysts the catalytic activity of AuNPs increases with increasing the amount of mesopores, leading to higher and faster D-glucose conversion (Figure S8). This is in agreement with previous studies where catalysis with deposited metal NPs requires a high surface area of the porous support because of sufficient dispersion of the active phase. ${ }^{41-44}$ However, all of the supports employed here have in general a high porosity, and the most active catalyst in this study is Au-CMK-3, suggesting that the presence, amount, and architecture of 
mesopores, together with the overall porosity, play a crucial role. Ordered mesoporous CMK-3 has pores of a narrow diameter distribution around $6 \mathrm{~nm}$, which are well interconnected without bottlenecks. Together with micropores present inside the hexagonally arranged carbon rods, this material has a hierarchical network of pores. On the other hand, the pores in STC materials are built based on the clustering of $\mathrm{ZnCl}_{2}$ as a salt porogen. This results in a porous system that is still interconnected but with a larger portion of pores that are less easily accessible for the solvent or the solute. In all the catalysts, the active phase is mainly located on the surface of the material, as supported by XPS measurements. The probability of AuNPs being deposited inside of the mesopores can be ruled out by the relation between porosity and TEM particle size analysis as discussed above. In view of the similar surface chemistry and comparable location and size of the AuNPs on the supports, it can be concluded that the local concentration of oxygen and D-glucose around AuNPs is a decisive parameter for the catalytic activity of these materials. Because of the broad distribution of mesopore diameters and low connectivity of the mesopores with the surface of the material, STCs can poorly contribute to the enhancement of oxygen and D-glucose dissolution, and thus the concentration of the reactants near the catalytically active sites remains limited. On the contrary, it appears that a large amount of uniform narrow mesopores connected to the surface of the catalyst, as in Au-CMK-3, leads to a nanoconfinement effect that significantly enhances the activity of the catalyst of the same particle size. For example, it is generally known that water that is under nanoconfinement shows higher gas solubility than bulk water. ${ }^{18,45-47}$ This fact increases the catalytic activity of this catalyst by increasing the concentration of the reactant in the proximity of the active sites. Such a "direct communication" between oxygen-enriched pores and AuNPs on the external particle surface is not present for the STC supports that suffer from a significant contribution of confined mesopores without unrestricted connection to the AuNPs. To point out that the catalytic activity of the gold nanoparticles of similar size and on support with similar chemical structure must be the same, we propose here to introduce the term "apparent catalytic activity" to make clear that the change in the speed of D-glucose oxidation does not arise from differences in the intrinsic properties of the AuNPs but is rather caused by changes of their local environment caused by differences in the textural properties of the carbon support materials.

In the context of these conclusions, it is of upmost importance to ensure that the catalytic steps solely occur on supported gold particles in proximity to the carbon pores and that the formation of unsupported AuNPs which could also be a source of catalytic activity can be ruled out. ${ }^{22,48}$ The metal leaching test of the reaction solution with ICP-OES reveals an $\mathrm{Au}$ concentration between 0.031 and $0.072 \mathrm{mg} \mathrm{L}^{-1}$ of $\mathrm{Au}$ in solution. This corresponds to less than $1 \%$ of the overall added $\mathrm{Au}$ and confirms that the catalysis is dominated by the supported AuNPs (Table S2). These results are in line with a recent study in which it has been shown that no glucose is converted anymore after filtration of carbon-supported gold catalysts comparable in structure to those reported here. ${ }^{39}$

It can be further seen from the absence of apparent catalytic activity for the purely microporous STC-1 that micropores do not contribute to this enhancement of the concentration of reactants near the catalytically active centers. This is likely caused by the limited wetting of such narrow pores without polar sites with the polar water molecules. In other words, the supply of reactants to the AuNPs in such a case depends solely on diffusion from the bulk solution. In contrast, the open mesopores in CMK-3 can serve as a reservoir of confined water with a high concentration of oxygen and D-glucose leading to a significantly enhanced apparent catalytic activity in D-glucose conversion for AuNPs of similar size on such a support.

Control experiments testing the hypothesis that water confined into mesopores of Au-CMK-3 acts as an oxygen reservoir were performed by saturating the dispersion of a catalyst with oxygen and subsequently starting the catalytic reaction without further addition of gas. Au-STC-8 and AuSTC-2 with disordered mesopores showed minor catalytic activity in the D-glucose oxidation reaction, whereas Au-CMK3 exhibited a steady formation of gluconic acid, as observed by the addition of $\mathrm{NaOH}$ (Figure 6). This indicates that a certain

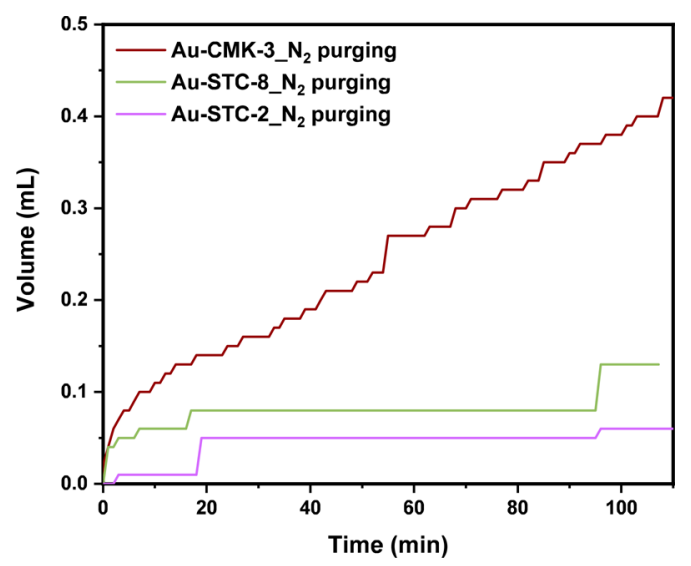

Figure 6. Volume of added $\mathrm{NaOH}$ vs time diagram for the reaction of D-glucose oxidation without the additional supply of oxygen during the reaction using the catalysts Au-STC-2, Au-STC-8, and Au-CMK3.

amount of oxygen is dissolved and stored in the pores of AuCMK-3, which enables oxidation of D-glucose even without a continued supply of oxygen to the reaction medium.

\section{CONCLUSION}

It has been shown that the pore architecture of carbon materials can have a crucial influence on the apparent catalytic activity of supported AuNPs. A series of carbons with different pore volumes and geometry in the micropore and mesopore range and comparable surface chemistry have been used, and the significant impact on the local concentration of reactants and thus on the apparent catalytic activity of AuNPs of comparable size was experimentally verified. Oxidation of $\mathrm{D}$ glucose with molecular oxygen in aqueous solution was applied as a model reaction in this study, with metal time yields ranging from no detectable activity when solely microporous support is used to $60.6 \mathrm{~mol}_{\mathrm{Glc}} \mathrm{min}^{-1} \mathrm{~mol}_{\mathrm{Au}}{ }^{-1}$ when ordered mesoporous support is used. However, the findings reported here have a general character, and they are limited to neither this particular reaction nor AuNPs or carbon support materials. When hydrophobic porous support materials are used in liquid phase catalysis with polar solvents, micropores may limit the supply of reactants to the active centers due to the limited wetting of narrow pores. This limitation can be overcome by the introduction of mesopores in the material. Nevertheless, 
this approach does not necessarily yield more active catalyst, as requirements of pore architecture and connectivity still need to be met. The present study shows that the open and ordered mesopores in hierarchical carbon provide a network for nanoconfined water, which, in turn, enriches educts in proximity to active sites, leading to higher apparent catalytic activity. The importance of the local environment of heterogeneous catalysts on their properties, which is of particular importance in liquid phase reactions has been exemplarily demonstrated for this system, and it is our belief that the nanostructure of support materials can be established as a future "regulation screw" to tailor the properties of supported nanoparticles in heterogeneous catalysis (in a similar sense as particle sizes and interaction between NPs and supports are already known for a long time).

\section{ASSOCIATED CONTENT}

\section{SI Supporting Information}

The Supporting Information is available free of charge at https://pubs.acs.org/doi/10.1021/acsanm.0c01299.

Thermogravimetric analysis results; comparison of metal time yields of catalytic reactions; Raman spectra; highresolution C 1s and O 1s XPS spectra; XPS table; TEM images of the catalysts after oxidation reaction; NMR spectra (PDF)

\section{AUTHOR INFORMATION}

\section{Corresponding Author}

Martin Oschatz - Department of Colloid Chemistry, MaxPlanck Institute of Colloids and Interfaces, 14476 Potsdam, Germany; Institute of Chemistry, University of Potsdam, 14476 Potsdam, Germany; 이이.org/0000-0003-2377-1214; Email: martin.oschatz@mpikg.mpg.de

\section{Authors}

Milena Perovic - Department of Colloid Chemistry, MaxPlanck Institute of Colloids and Interfaces, 14476 Potsdam, Germany

Nadezda V. Tarakina - Department of Colloid Chemistry, Max-Planck Institute of Colloids and Interfaces, 14476 Potsdam, Germany; iorcid.org/0000-0002-2365-861X

Jan Philipp Hofmann - Surface Science Laboratory, Department of Materials and Earth Sciences, Technische Universität Darmstadt, 64287 Darmstadt, Germany; Laboratory for Inorganic Materials and Catalysis, Department of Chemical Engineering and Chemistry, Eindhoven University of Technology, $5600 \mathrm{MB}$ Eindhoven, The Netherlands; (1) orcid.org/0000-0002-5765-1096

Complete contact information is available at: https://pubs.acs.org/10.1021/acsanm.0c01299

\section{Notes}

The authors declare no competing financial interest.

\section{ACKNOWLEDGMENTS}

The authors kindly acknowledge contributions from our colleagues at the Max Planck Institute of Colloids and Interfaces. Many thanks to Bolortuya Badamdorj (HRTEM), Rona Pitschke, Heike Runge (TEM), Antje Völkel (TGA), and Ines Below-Lutz (ICP-OES). We gratefully acknowledge financial support by the Max Planck Society and by the Deutsche Forschungsgemeinschaft (DFG, German Research
Foundation) under Germany's Excellence Strategy-EXC 2008/1-390540038.

\section{REFERENCES}

(1) den Breejen, J. P.; Radstake, P. B.; Bezemer, G. L.; Bitter, J. H.; Frøseth, V.; Holmen, A.; de Jong, K. P. On the Origin of the Cobalt Particle Size Effects in Fischer-Tropsch Catalysis. J. Am. Chem. Soc. 2009, 131 (20), 7197-7203.

(2) Stratakis, M.; Garcia, H. Catalysis by Supported Gold Nanoparticles: Beyond Aerobic Oxidative Processes. Chem. Rev. 2012, 112 (8), 4469-4506.

(3) Schauermann, S.; Nilius, N.; Shaikhutdinov, S.; Freund, H.-J. Nanoparticles for Heterogeneous Catalysis: New Mechanistic Insights. Acc. Chem. Res. 2013, 46 (8), 1673-1681.

(4) Raimondi, F.; Scherer, G. G.; Kötz, R.; Wokaun, A. Nanoparticles in Energy Technology: Examples from Electrochemistry and Catalysis. Angew. Chem., Int. Ed. 2005, 44 (15), 2190-2209.

(5) Torres Galvis, H. M.; Bitter, J. H.; Khare, C. B.; Ruitenbeek, M.; Dugulan, A. I.; de Jong, K. P. Supported Iron Nanoparticles as Catalysts for Sustainable Production of Lower Olefins. Science 2012, 335 (6070), 835-838.

(6) Cuenya, B. R. Synthesis and catalytic properties of metal nanoparticles: Size, shape, support, composition, and oxidation state effects. Thin Solid Films 2010, 518 (12), 3127-3150.

(7) Mavrikakis, M.; Hammer, B.; Nørskov, J. K. Effect of Strain on the Reactivity of Metal Surfaces. Phys. Rev. Lett. 1998, 81 (13), 28192822.

(8) Bianchi, C.; Porta, F.; Prati, L.; Rossi, M. Selective liquid phase oxidation using gold catalysts. Top. Catal. 2000, 13 (3), 231-236.

(9) Bezemer, G. L.; Bitter, J. H.; Kuipers, H. P. C. E.; Oosterbeek, H.; Holewijn, J. E.; Xu, X.; Kapteijn, F.; van Dillen, A. J.; de Jong, K. P. Cobalt Particle Size Effects in the Fischer-Tropsch Reaction Studied with Carbon Nanofiber Supported Catalysts. J. Am. Chem. Soc. 2006, 128 (12), 3956-3964.

(10) White, R. J.; Luque, R.; Budarin, V. L.; Clark, J. H.; Macquarrie, D. J. Supported metal nanoparticles on porous materials. Methods and applications. Chem. Soc. Rev. 2009, 38 (2), 481-494.

(11) Gerber, I. C.; Serp, P. A Theory/Experience Description of Support Effects in Carbon-Supported Catalysts. Chem. Rev. 2020, 120 (2), 1250-1349.

(12) Ishida, T.; Murayama, T.; Taketoshi, A.; Haruta, M. Importance of Size and Contact Structure of Gold Nanoparticles for the Genesis of Unique Catalytic Processes. Chem. Rev. 2020, 120 (2), 464-525.

(13) Qin, Q.; Heil, T.; Antonietti, M.; Oschatz, M. Single-Site Gold Catalysts on Hierarchical N-Doped Porous Noble Carbon for Enhanced Electrochemical Reduction of Nitrogen. Small Methods 2018, 2 (12), 1800202.

(14) Oschatz, M.; Hofmann, J. P.; van Deelen, T. W.; Lamme, W. S.; Krans, N. A.; Hensen, E. J. M.; de Jong, K. P. Effects of the Functionalization of the Ordered Mesoporous Carbon Support Surface on Iron Catalysts for the Fischer-Tropsch Synthesis of Lower Olefins. ChemCatChem 2017, 9 (4), 620-628.

(15) Farmer, J. A.; Campbell, C. T. Ceria Maintains Smaller Metal Catalyst Particles by Strong Metal-Support Bonding. Science 2010, 329 (5994), 933-936.

(16) Hu, J.; Chen, L.; Zhu, K.; Suchopar, A.; Richards, R. Aerobic oxidation of alcohols catalyzed by gold nano-particles confined in the walls of mesoporous silica. Catal. Today 2007, 122 (3), 277-283.

(17) Xiao, J.; Pan, X.; Guo, S.; Ren, P.; Bao, X. Toward Fundamentals of Confined Catalysis in Carbon Nanotubes. J. Am. Chem. Soc. 2015, 137 (1), 477-482.

(18) Lidon, P.; Marker, S. C.; Wilson, J. J.; Williams, R. M.; Zipfel, W. R.; Stroock, A. D. Enhanced Oxygen Solubility in Metastable Water under Tension. Langmuir 2018, 34 (40), 12017-12024.

(19) Dimitratos, N.; Lopez-Sanchez, J. A.; Hutchings, G. J. Selective liquid phase oxidation with supported metal nanoparticles. Chem. Sci. 2012, 3 (1), 20-44. 
(20) Biella, S.; Prati, L.; Rossi, M. Selective Oxidation of D-Glucose on Gold Catalyst. J. Catal. 2002, 206 (2), 242-247.

(21) Okatsu, H.; Kinoshita, N.; Akita, T.; Ishida, T.; Haruta, M. Deposition of gold nanoparticles on carbons for aerobic glucose oxidation. Appl. Catal., A 2009, 369 (1), 8-14.

(22) Comotti, M.; Della Pina, C.; Matarrese, R.; Rossi, M. The Catalytic Activity of "Naked" Gold Particles. Angew. Chem., Int. Ed. 2004, 43 (43), 5812-5815.

(23) Önal, Y.; Schimpf, S.; Claus, P. Structure sensitivity and kinetics of $\mathrm{D}$-glucose oxidation to $\mathrm{D}$-gluconic acid over carbon-supported gold catalysts. J. Catal. 2004, 223 (1), 122-133.

(24) Mirescu, A.; Berndt, H.; Martin, A.; Prüße, U. Long-term stability of a $0.45 \% \mathrm{Au} / \mathrm{TiO}_{2}$ catalyst in the selective oxidation of glucose at optimized reaction conditions. Appl. Catal., A 2007, 317 (2), 204-209.

(25) Jun, S.; Joo, S. H.; Ryoo, R.; Kruk, M.; Jaroniec, M.; Liu, Z.; Ohsuna, T.; Terasaki, O. Synthesis of New, Nanoporous Carbon with Hexagonally Ordered Mesostructure. J. Am. Chem. Soc. 2000, 122 (43), 10712-10713.

(26) Fechler, N.; Fellinger, T.-P.; Antonietti, M. Salt Templating”: A Simple and Sustainable Pathway toward Highly Porous Functional Carbons from Ionic Liquids. Adv. Mater. 2013, 25 (1), 75-79.

(27) Yan, R.; Antonietti, M.; Oschatz, M. Toward the Experimental Understanding of the Energy Storage Mechanism and Ion Dynamics in Ionic Liquid Based Supercapacitors. Adv. Energy Mater. 2018, 8 (18), 1800026.

(28) Zhao, D.; Feng, J.; Huo, Q.; Melosh, N.; Fredrickson, G. H.; Chmelka, B. F.; Stucky, G. D. Triblock Copolymer Syntheses of Mesoporous Silica with Periodic 50 to 300 Angstrom Pores. Science 1998, 279 (5350), 548-552.

(29) Turkevich, J.; Stevenson, P. C.; Hillier, J. A study of the nucleation and growth processes in the synthesis of colloidal gold. Discuss. Faraday Soc. 1951, 11 (0), 55-75.

(30) Armstrong, R. D.; Hirayama, J.; Knight, D. W.; Hutchings, G. J. Quantitative Determination of Pt- Catalyzed d-Glucose Oxidation Products Using 2D NMR. ACS Catal. 2019, 9 (1), 325-335.

(31) Lu, A.-H.; Schüth, F. Nanocasting: A Versatile Strategy for Creating Nanostructured Porous Materials. Adv. Mater. 2006, 18 (14), 1793-1805.

(32) Thommes, M.; Kaneko, K.; Neimark, A. V.; Olivier, J. P.; Rodriguez-Reinoso, F.; Rouquerol, J.; Sing, K. S. W. Physisorption of gases, with special reference to the evaluation of surface area and pore size distribution (IUPAC Technical Report). Pure Appl. Chem. 2015, 87, 1051-1069.

(33) Casiraghi, C.; Ferrari, A. C.; Robertson, J. Raman spectroscopy of hydrogenated amorphous carbons. Phys. Rev. B: Condens. Matter Mater. Phys. 2005, 72 (8), 085401.

(34) Ferrari, A. C.; Robertson, J. Interpretation of Raman spectra of disordered and amorphous carbon. Phys. Rev. B: Condens. Matter Mater. Phys. 2000, 61 (20), 14095-14107.

(35) Oschatz, M.; Pré, P.; Dörfler, S.; Nickel, W.; Beaunier, P.; Rouzaud, J.-N.; Fischer, C.; Brunner, E.; Kaskel, S. Nanostructure characterization of carbide-derived carbons by morphological analysis of transmission electron microscopy images combined with physisorption and Raman spectroscopy. Carbon 2016, 105, 314-322.

(36) Casavola, M.; Hermannsdörfer, J.; de Jonge, N.; Dugulan, A. I.; de Jong, K. P. Fabrication of Fischer-Tropsch Catalysts by Deposition of Iron Nanocrystals on Carbon Nanotubes. Adv. Funct. Mater. 2015, 25 (33), 5309-5319.

(37) Lama, S. M. G.; Schmidt, J.; Malik, A.; Walczak, R.; Silva, D. V.; Völkel, A.; Oschatz, M. Modification of salt-templated carbon surface chemistry for efficient oxidation of glucose with supported gold catalysts. ChemCatChem 2018, 10, 2458.

(38) Galeano, C.; Meier, J. C.; Peinecke, V.; Bongard, H.; Katsounaros, I.; Topalov, A. A.; Lu, A.; Mayrhofer, K. J. J.; Schüth, F. Toward Highly Stable Electrocatalysts via Nanoparticle Pore Confinement. J. Am. Chem. Soc. 2012, 134 (50), 20457-20465.

(39) Perazzolo, V.; Brandiele, R.; Durante, C.; Zerbetto, M.; Causin, V.; Rizzi, G. A.; Cerri, I.; Granozzi, G.; Gennaro, A. Density
Functional Theory (DFT) and Experimental Evidences of MetalSupport Interaction in Platinum Nanoparticles Supported on Nitrogen- and Sulfur-Doped Mesoporous Carbons: Synthesis, Activity, and Stability. ACS Catal. 2018, 8 (2), 1122-1137.

(40) Casaletto, M. P.; Longo, A.; Martorana, A.; Prestianni, A.; Venezia, A. M. XPS study of supported gold catalysts: the role of Au0 and $\mathrm{Au}+\delta$ species as active sites. Surf. Interface Anal. 2006, 38 (4), $215-218$.

(41) Qi, P.; Chen, S.; Chen, J.; Zheng, J.; Zheng, X.; Yuan, Y. Catalysis and Reactivation of Ordered Mesoporous CarbonSupported Gold Nanoparticles for the Base-Free Oxidation of Glucose to Gluconic Acid. ACS Catal. 2015, 5 (4), 2659-2670.

(42) Ishida, T.; Kinoshita, N.; Okatsu, H.; Akita, T.; Takei, T.; Haruta, M. Influence of the support and the size of gold clusters on catalytic activity for glucose oxidation. Angew. Chem., Int. Ed. 2008, 47 (48), 9265-9268.

(43) Xiong, H.; Jewell, L. L.; Coville, N. J. Shaped Carbons As Supports for the Catalytic Conversion of Syngas to Clean Fuels. ACS Catal. 2015, 5 (4), 2640-2658.

(44) Bianchi, C. B.; Biella, S.; Gervasini, A.; Prati, L.; Rossi, M. Gold on carbon: influence of support properties on catalyst activity in liquid-phase oxidation. Catal. Lett. 2003, 85, 91-96.

(45) Soubeyrand-Lenoir, E.; Vagner, C.; Yoon, J. W.; Bazin, P.; Ragon, F.; Hwang, Y. K.; Serre, C.; Chang, J.-S.; Llewellyn, P. L. How Water Fosters a Remarkable 5-Fold Increase in Low-Pressure $\mathrm{CO}_{2}$ Uptake within Mesoporous MIL-100(Fe). J. Am. Chem. Soc. 2012, 134 (24), 10174-10181.

(46) Pera-Titus, M.; El-Chahal, R.; Rakotovao, V.; Daniel, C.; Miachon, S.; Dalmon, J.-A. Direct Volumetric Measurement of Gas Oversolubility in Nanoliquids: Beyond Henry's Law. ChemPhysChem 2009, 10 (12), 2082-2089.

(47) Luzar, A.; Bratko, D. Gas Solubility in Hydrophobic Confinement. J. Phys. Chem. B 2005, 109 (47), 22545-22552.

(48) Oliver-Meseguer, J.; Cabrero-Antonino, J. R.; Domínguez, I.; Leyva-Pérez, A.; Corma, A. Small Gold Clusters Formed in Solution Give Reaction Turnover Numbers of $10^{7}$ at Room Temperature. Science 2012, 338 (6113), 1452-1455. 